\title{
Quantum kagomé antiferromagnet in a magnetic field: Low-lying nonmagnetic excitations versus valence-bond crystal order
}

\author{
D. C. Cabra, ${ }^{1}$ M. D. Grynberg, ${ }^{2}$ P. C. W. Holdsworth, ${ }^{3}$ A. Honecker, ${ }^{4,5}$ P. Pujol,,${ }^{3}$ J. Richter, ${ }^{6}$ D. Schmalfuß,${ }^{6}$ and \\ J. Schulenburg ${ }^{7}$ \\ ${ }^{1}$ Université Louis Pasteur, Laboratoire de Physique Théorique, 67084 Strasbourg Cédex, France \\ ${ }^{2}$ Departamento de Física, Universidad Nacional de La Plata, C.C. 67, (1900) La Plata, Argentina \\ ${ }^{3}$ Laboratoire de Physique, ENS Lyon, 46 Allée d'Italie, 69364 Lyon Cédex 07, France \\ ${ }^{4}$ TU Braunschweig, Institut für Theoretische Physik, 38106 Braunschweig, Germany \\ ${ }^{5}$ Universität Hannover, Institut für Theoretische Physik, Appelstrasse 2, 30167 Hannover, Germany \\ ${ }^{6}$ Institut für Theoretische Physik, Otto-von-Guericke Universität Magdeburg, 39016 Magdeburg, Germany \\ ${ }^{7}$ Universitätsrechenzentrum, Otto-von-Guericke-Universität Magdeburg, 39016 Magdeburg, Germany
}

(Received 23 December 2004; published 27 April 2005)

\begin{abstract}
We study the ground state properties of a quantum antiferromagnet on the kagomé lattice in the presence of a magnetic field, paying particular attention to the stability of the plateau at magnetization $1 / 3$ of saturation and the nature of its ground state. We discuss fluctuations around classical ground states and argue that quantum and classical calculations at the harmonic level do not lead to the same result in contrast to the zero-field case. For spin $S=1 / 2$ we find a magnetic gap below which an exponential number of nonmagnetic excitations are present. Moreover, such non-magnetic excitations also have a (much smaller) gap above the threefold degenerate ground state. We provide evidence that the ground state has long-range order of valencebond crystal type with nine spins in the unit cell.
\end{abstract}

DOI: $10.1103 /$ PhysRevB.71.144420

PACS number(s): 75.10.Jm, 75.45.+j, 75.60.Ej

\section{INTRODUCTION}

The appearance of exotic quantum phases in systems described by two-dimensional frustrated antiferromagnets is presently the subject of intense research (see, e.g., Refs. 1 and 2 for recent reviews). The Heisenberg antiferromagnet on highly frustrated lattices such as the pyrochlore and kagomé lattice has a huge degeneracy of the classical ground state such that no magnetic order arises at any temperature (see, e.g., Ref. 3 for a recent review). At the quantum level one may then obtain different exotic phases without magnetic (Néel) order. One such phase is the so-called "valencebond crystal," which is characterized by formation of local singlets in a long-range ordered pattern. An even more exotic phase, namely, one without any kind of long-range order, is suspected to arise in the $S=1 / 2$ Heisenberg model on the kagomé lattice. ${ }^{1,4-7}$ In the latter case, there is a small spin gap and, although this is still under discussion, ${ }^{8,9}$ the ground state is suspected to be disordered. In particular, a huge number of singlets (exponentially growing with the system size) is found inside the spin gap, which is reminiscent of the classical degeneracy.

The spin $S=1 / 2$ kagomé Heisenberg antiferromagnet (KHAFM) is realized, e.g., in volborthite, ${ }^{10}$ although presumably in some distorted form. Another possible realization is given by atomic quantum gases in optical lattices. ${ }^{11}$ In the latter case, magnetization corresponds to particle number and a magnetic field to chemical potential, opening the possibility to perform experiments for the behavior of the spin model in a magnetic field.

The magnetization process of the KHAFM has been studied theoretically both for classical ${ }^{12-14}$ as well as quantum spins. ${ }^{14-17}$ Numerical results for the magnetization curve of the $S=1 / 2$ Heisenberg model exhibit, among others, a clear plateau at $1 / 3$ of the saturation magnetization ${ }^{14-17}$ (see also Fig. 1). For the classical KHAFM at one third of the saturation field thermal fluctuations select collinear states, but there appears to be no real order. ${ }^{12}$ For the $S=1 / 2$ KHAFM, we will argue in this paper that the state with magnetization $\langle M\rangle=1 / 3$ exhibits order of the valence-bond crystal type (the spin-spin correlation functions are short ranged ${ }^{18}$ ), although it shares some similarities with the case $\langle M\rangle=0$.

In the present paper we study the $X X Z$ model in a magnetic field $h$, given by

$$
H=J \sum_{\langle i, j\rangle}\left(s_{i}^{x} s_{j}^{x}+s_{i}^{y} s_{j}^{y}+\Delta s_{i}^{z} s_{j}^{z}\right)-h \sum_{i} s_{i}^{z},
$$

where $\langle i, j\rangle$ indicates nearest neighbors on the kagomé lattice (see inset of Fig. 1), $s_{i}^{\alpha}$ are spin-half operators acting at site $i$ and $\Delta$ is the $X X Z$ anisotropy.

\section{EXACT DIAGONALIZATION FOR THE $S=1 / 2$ HEISENBERG MODEL}

First we present numerical results that have been obtained by Lanczos diagonalization of the Hamiltonian (1) using the program package SPINPACK. ${ }^{19}$ All numerical computations have been performed on lattices with $N$ sites subject to periodic boundary conditions.

The main panel of Fig. 1 shows the boundaries of the fully polarized state (which we normalize to $\langle M\rangle=1$ ) and a state with $\langle M\rangle=1 / 3$ in the $X X Z$ model (1). Figure 2(a) shows the low-lying excitations above the $\langle M\rangle=1 / 3$ ground state computed by exact diagonalization for $N=36$ and $\Delta=1$. The dashed lines show the gap to states with $S^{z}=5$ and 7, which 


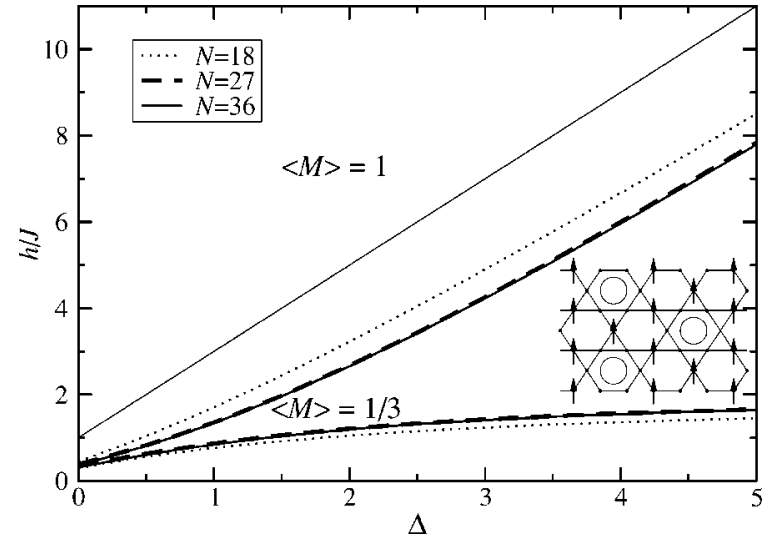

FIG. 1. Boundaries of the $\langle M\rangle=1 / 3$ plateau as a function of the anisotropy $\Delta$ for different lattice sizes (see legend) and the transition to saturation $\langle M\rangle=1$ for the thermodynamic limit (thin full line). Inset: kagomé lattice with an ordered state of the valencebond crystal type at $\langle M\rangle=1 / 3$ : circles in certain hexagons indicate local resonances between different Néel configurations on the hexagons, arrows indicate spins which are aligned with the field.

vanishes at the boundaries of the plateau. The maximum magnetic gap occurs in the middle of the plateau where these two lines intersect, and we will use this as a definition of the magnetic gap. Horizontal straight lines denote states with $S^{z}=6$ (i.e., $\langle M\rangle=1 / 3$ ) and correspond to nonmagnetic excitations. The large number of nonmagnetic excitations below the magnetic gap is reminiscent of the classical degeneracy. The shape of the integrated density of nonmagnetic excitations [see Fig. 2(b)] is very similar to the corresponding integrated density of singlets at $\langle M\rangle=0$ (see Fig. 2 of Ref. 5). In combination with the disordered classical ground state, ${ }^{12}$ one might be tempted to take this as evidence that the ground state of the $S=1 / 2$ KHAFM at $\langle M\rangle=1 / 3$ is also disordered.

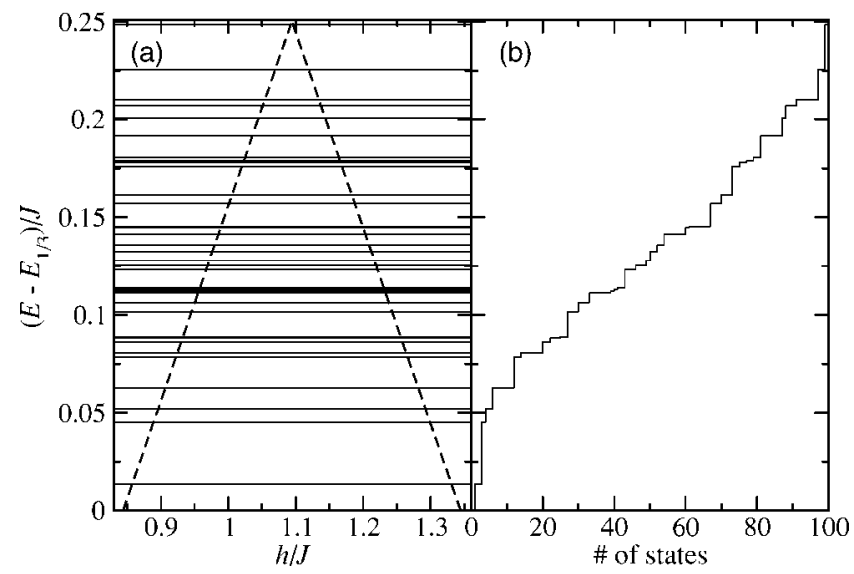

FIG. 2. Low-lying excitations above the $\langle M\rangle=1 / 3$ plateau for the $S=1 / 2$ Heisenberg antiferromagnet on the $N=36$ kagomé lattice. (a) Full lines show all excitations with $S^{z}=6$ in the given energy range, bold dashed lines the lowest excitations with $S^{z}=5$ and $S^{z}=7$ as a function of magnetic field $h$. (b) Excitation energy versus number of states with $S^{z}=6$ below that energy. One observes a total of 100 states below the magnetic gap in the middle of the $\langle M\rangle$ $=1 / 3$ plateau (corresponding to the largest gap to magnetic excitations).
However, we will argue next that here classical and quantum fluctuations are in fact not equivalent at the harmonic level, and will then present evidence in favor of an ordered state for $S=1 / 2$.

\section{FLUCTUATIONS AROUND THE CLASSICAL GROUND STATE}

Classical (thermal) fluctuations were studied in Refs. 12, 14 , and 20 such that we make only a few comments valid for nonzero magnetization and arbitrary anisotropy $\Delta$. As was shown explicitly for $\Delta=1$ in Ref. 12, thermal fluctuations select collinear "up-up-down" (UUD) configurations at $\langle M\rangle$ $=1 / 3$ against the other noncollinear configurations that also minimize the classical energy, but all UUD configurations have the same spectra of harmonic fluctuations. Indeed, a local change of variables shows that the covering-dependent Hamiltonians of classical Gaussian fluctuations ${ }^{14}$ are equivalent.

The role of quantum fluctuations is, however, radically different. Now, quantum commutation relations have to be preserved and the change of variables used for the classical case is no longer possible. To analyze this in more detail, we compute the zero-point contribution to the ground state energy at $\langle M\rangle=1 / 3$ for two different coverings with $q=0$ and a $\sqrt{3} \times \sqrt{3}$ structure, respectively (the latter can be found, e.g., in Fig. 1 of Ref. 12). By writing the spin operators on each site in terms of bosonic creation and annihilation operators, as

$$
\vec{s}_{l}=S\left(\frac{1}{\sqrt{2 S}}\left(a_{l}^{\dagger}+a_{l}\right), i \frac{1}{\sqrt{2 S}}\left(a_{l}^{\dagger}-a_{l}\right), 1-\frac{a_{l}^{\dagger} a_{l}}{S}\right),
$$

we obtain the Hamiltonian

$$
H=H_{0}+\frac{S}{2}\left[H_{2}+O(1 / \sqrt{S})\right],
$$

where $\mathrm{H}_{2}$ is quadratic in creation and annihilation operators and the $O(1 / \sqrt{S})$ part contains higher orders. By Fourier transforming, we obtain

$$
H_{2}=\frac{J}{2} \sum_{\vec{k}}\left(a_{-\vec{k}}^{\dagger i}, a_{\vec{k}}^{i}\right) \cdot\left(\begin{array}{cc}
\tilde{M}^{+} & \tilde{M}^{-} \\
\tilde{M}^{-} & \tilde{M}^{+}
\end{array}\right)_{i j} \cdot\left(\begin{array}{c}
a_{-\vec{k}}^{j} \\
a_{\vec{k}}^{\dagger j}
\end{array}\right),
$$

where $\tilde{M}^{ \pm}$are $3 \times 3$ and $9 \times 9$ matrices for the $q=0$ and the $\sqrt{3} \times \sqrt{3}$ states since these coverings have three and nine sublattices, respectively. No further change of variables is possible here since the commutation relations of the su(2) algebra of the spins have to be preserved. At $\Delta=1$ one finds for the zero-point fluctuations $\frac{1}{2} \sum_{\vec{k}} \omega_{\vec{k}}=J S / 3$ and $\approx 0.5643 J S$ for the $q=0$ and the $\sqrt{3} \times \sqrt{3}$ state, respectively, demonstrating the inequivalence of the different coverings at the quantum level.

\section{EFFECTIVE MODEL FOR THE ISING LIMIT}

Let us now return to the extreme quantum case $S=1 / 2$ and study the anisotropic $X X Z$ limit. For $\Delta \gg 1$, we can adapt 
the analysis of Refs. 21 and 22 of the Ising model in a transverse field to the $X X Z$ model by replacing the expansion in the transverse field with an expansion in powers of $1 / \Delta$.

In the Ising limit $\Delta=\infty$, the ground states are those states in which around each triangle, two spins point up and one down. This ground state space of the Ising model can then be taken as configuration space for a perturbative treatment of the $X Y$ part of the $X X Z$ Hamiltonian.

For sufficiently large lattices, the lowest nontrivial order is third order, flipping simultaneously pairwise antiparallel spins around a hexagon. This is described by an effective Hamiltonian ${ }^{21,22}$

$$
H_{\text {eff }}=\lambda \sum_{\text {hexagon } i}\left(s_{i, 1}^{+} s_{i, 2}^{-} s_{i, 3}^{+} s_{i, 4}^{-} s_{i, 5}^{+} s_{i, 6}^{-}+s_{i, 1}^{-} s_{i, 2}^{+} s_{i, 3}^{-} s_{i, 4}^{+} s_{i, 5}^{-} s_{i, 6}^{+}\right),
$$

where the spin operator $s_{i, j}^{\alpha}$ operates at the $j$ th site around hexagon $i$ and $\lambda=3 J /\left(2 \Delta^{2}\right)$. Note that in the Isingbasis the effective Hamiltonian (5) has only off-diagonal matrix elements of size $\lambda$.

The configurations of the Ising model can be mapped to dimer coverings of the dual lattice which in the case of the kagomé lattice is the hexagonal lattice. Now one can use known results for dimer coverings ${ }^{23,24}$ to write down the asymptotic growth law for the number of Ising configurations $\mathcal{N}_{\text {conf }}$ on an $N$-site kagomé lattice:

$$
\mathcal{N}_{\text {conf }} \propto(1.11372781 \ldots)^{N} .
$$

Exploiting results for the related quantum dimer model on the hexagonal lattice, ${ }^{25}$ Moessner and Sondhi concluded ${ }^{22}$ that the ground state of the effective Hamiltonian (5) is of the valence-bond crystal type. To be more precise, the case studied in Refs. 22 and 25 corresponds to $\lambda<0$, whereas we have $\lambda>0$, but there exist unitary transformations which change the sign of $\lambda .{ }^{26}$ Hence, the spectra of the effective Hamiltonian (5) are invariant under $\lambda \rightarrow-\lambda$. The three-fold degenerate ground-state wave functions are sketched in the inset of Fig. 1. Circles in one third of the hexagons denote resonances between the two different Néel states on the surrounding hexagon; a background of the remaining third of all spins points in the direction of the field. Note that these wave functions were argued in ${ }^{22,25}$ to yield a qualitatively correct description, but they should not be used for a quantitative analysis. Furthermore, we emphasize that due to the resonances, these wave functions are of a purely quantum nature and have no counterparts as unique states of the classical Heisenberg model.

According to the above, at large $\Delta$ the $\langle M\rangle=1 / 3$ state of the $X X Z$ model on the kagomé lattice should be threefold degenerate with a gap to the next nonmagnetic excitations. To check this conclusion and compare it to Fig. 2, let us look at the spectrum of the effective Hamiltonian (5). This effective model has a substantially reduced Hilbert space (e.g., for $N=36$ there are only 120 states). We can therefore go to larger lattice sizes than in the full model. Results for kagomé lattices with up to $N=144$ sites are shown in Fig. 3. Additional short cycles wrap around the boundaries of the lattice for $N \leqslant 27$ and lead to nongeneric ground states of $H_{\text {eff. }}$ Ac-

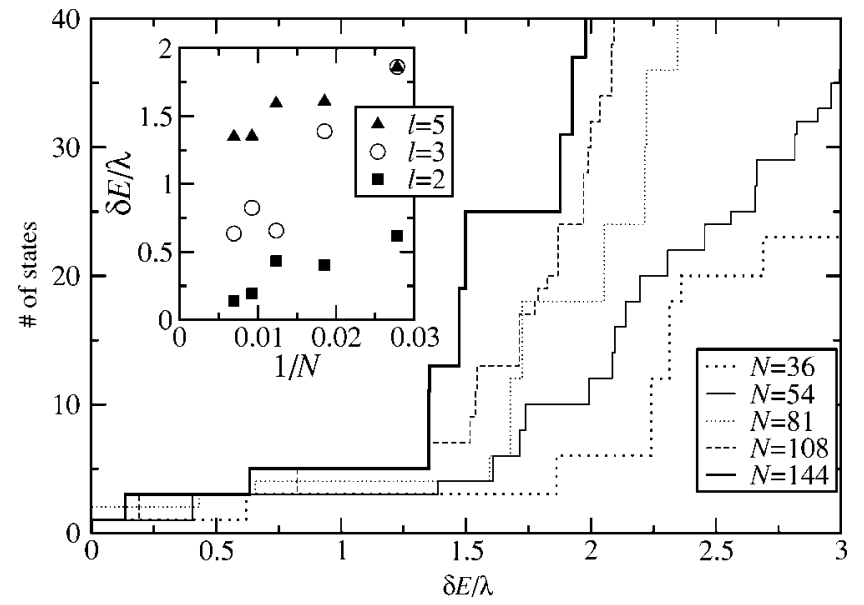

FIG. 3. Main panel: Spectra of the effective Hamiltonian (5) for $\Delta \rightarrow \infty$ with $N=36,54,81,108$, and 144 . Inset: Scaling of the energy of the $l$ th excited state with inverse system size $1 / N$ for some selected levels.

cordingly, systems with $N<36$ should not be considered and are not included in Fig. 3.

Two features are apparent in Fig. 3, at least for the two biggest system sizes ( $N=108$ and 144). Firstly, there are two further levels above the ground state. The finite-size dependence of the second excited state is shown by $l=2$ in the inset of Fig. 3 and indicates that it is converging to $\delta E \rightarrow 0$, which is consistent with the expected threefold degeneracy of the ground state in the thermodynamic limit. Secondly, there is a huge density of states emerging for $\delta E \geqslant 1.3 \lambda$. The finite-size behavior of the $l=5$ level in the inset of Fig. 3 indicates that a gap of the order $\sim 1.2 \lambda$ to these higher excited states persists in the thermodynamic limit. For $N=108$ and 144 there are two further levels in between. It is difficult to extrapolate their energies to $N \rightarrow \infty$, but the behavior of the $l=3$ excited level in the inset of Fig. 3 at the largest values of $N$ suggests that they retain a finite gap in the thermodynamic limit. Inspection of the wave-functions indicates that these additional low-lying levels may arise from the three classical $\sqrt{3} \times \sqrt{3}$ configurations.

\section{FROM THE ISING LIMIT TO THE HEISENBERG MODEL}

Although the effective Hamiltonian leads to higher degeneracies of some excited states, the $N=36$ curves in Figs. 2 and 3 have a very similar shape, which can be taken as a first indication that the same scenario as for $\Delta \gg 1$ also applies to $\Delta=1$. Comparison of the overall scales leads to an estimate for the gap in the $\langle M\rangle=1 / 3$ sector at $\Delta=1$ of about $0.04 \mathrm{~J}$. Furthermore, the total number of Ising configurations is very close to the number of nonmagnetic excitations below the magnetic gap for $\Delta=1$ at a given system size (see also Ref. 18). Hence, the growth law (6) yields a good approximation also to the number of nonmagnetic excitations in the Heisenberg model $(\Delta=1)$.

It is instructive to compute the overlap of the wave function of the full $X X Z$ model, $\mid$ full $X X Z\rangle$, with the ground state 


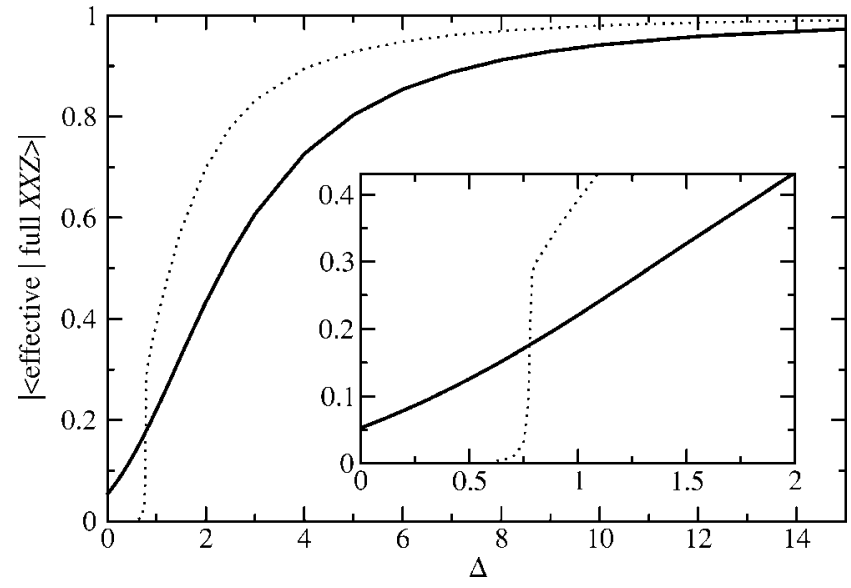

FIG. 4. Overlap between the ground state of the full $X X Z$ model and the effective Hamiltonian with $N=36$ for different values of the anisotropy parameter $\Delta$ (full line). For comparison, we include the corresponding result for a different model; namely, the $N=36$ triangular lattice (Ref. 17) (dotted line), which exhibits a sharp drop around $\Delta=0.77$.

wave function, |effective $\rangle$, of the effective Hamiltonian with the same number of spins $N$. The analysis of the effective Hamiltonian implies that one should study only sizes that are multiples of nine, and that $N=36$ is the smallest size which is representative of the general case. However, $N=36$ is the biggest system in which we have been able to study the full $X X Z$ model and hence is the only case we can discuss. Results for the overlap $\mid\langle$ effective $|$ full $X X Z\rangle \mid$ are shown by the full line in Fig. 4. We observe that this overlap tends to 1 for large values of $\Delta$, as expected. Furthermore, the overlap remains appreciable even close to the Heisenberg model $(\mid\langle$ effective $|$ full $X X Z\rangle \mid \approx 0.22$ for $\Delta=1)$, in particular if one considers that the dimension of the symmetry subspace under consideration is of the order $10^{7}$. Note further that an analogous computation of the overlap of the ground state of the Ising and the full $X X Z$ model at $\langle M\rangle=1 / 3$ on the triangular lattice leads to a sharp drop at $\Delta \approx 0.76$ for a fixed $N$ (see dotted curve in Fig. 4 for $N=36$ ), signaling an instability of the plateau state. ${ }^{17}$ No such sharp drop is observed on the kagomé lattice (full line in Fig. 4), which we take as a sign of absence of phase transitions between $\Delta=\infty$ and $\approx 0$ in the $X X Z$ model on the kagomé lattice at $\langle M\rangle=1 / 3$. In particular, $\Delta=1$ and $\infty$ should belong to the same phase.

\section{DISCUSSION AND CONCLUSIONS}

To conclude, we have analyzed the low-energy spectrum of the kagomé $X X Z S=1 / 2$ model at magnetization $\langle M\rangle$
$=1 / 3$. While the existence of a magnetization plateau is clear, the nature of the nonmagnetic excitations over the ground state is more difficult to clarify. We have argued by different techniques that the ground state has an order of the valence-bond crystal type; i.e., the ground state is threefold degenerate and there is a small gap to all higher excitations. While in the case $\Delta \gg 1$ this scenario is derived from a mapping to an effective Hamiltonian, ${ }^{21,22,25}$ our numerical data indicate that it persists down to the isotropic limit $\Delta=1$.

One of the key differences between the present case and $\langle M\rangle=0$ lies in the unrenormalized classical thermal and quantum fluctuations. In the absence of a magnetic field, they are equivalent regarding the lifting of degeneracy of configurations with soft modes (planar configurations). However, for $\langle M\rangle=1 / 3$, classical thermal fluctuations select the collinear UUD configurations and the weight in the free energy of any UUD covering is equivalent at the harmonic level. Because of commutation relations that have to be preserved at the quantum level, the zero-point corrections over the UUD configurations are no longer equal. This is the first indication that a spin liquid phase is less likely to appear than for the $\langle M\rangle=0$ case.

For $S=1 / 2$ and $\Delta=1$ we find, for $\langle M\rangle=1 / 3$, an exponential number of nonmagnetic excitations below the magnetic gap that are reminiscent of the classical degeneracy; just as for $\langle M\rangle=0 .{ }^{1,5,6}$ In the latter case the macroscopic number of nonmagnetic excitations has been taken as evidence for a completely disordered ground state. Here, however, we find evidence for a further small gap, separating the continuum of states from a ground state, which has long-range order of valence-bond crystal type. We remark that the $N=36$ spectrum $^{5}$ suggests that candidates for valence-bond ordered states for $\langle M\rangle=0$ would have a larger unit cell than the state above. Hence, we believe that the issue of order at very low energies in the $S=1 / 2 \mathrm{KHAFM}$ at $\langle M\rangle=0$ remains a challenging problem.

\section{ACKNOWLEDGMENTS}

We would like to thank the Rechenzentrum, TU Braunschweig for allocation of CPU time on the compute-server CFGAUSS and J. Schüle for technical support. We are grateful to H.-U. Everts, C. Lhuillier, F. Mila, and R. Moessner for useful discussions. This work was financially supported by ECOS-Sud and Procope-Egide exchange programs as well as by the Deutsche Forschungsgemeinschaft (Project Ri615/121). The research of D.C.C. and M.D.G. was partially supported by CONICET and Fundación Antorchas, Argentina (Grant No. A-13622/1-106).
${ }^{1}$ G. Misguich and C. Lhuillier, in Frustrated Spin Systems, edited by H. T. Diep (World Scientific, Singapore, 2004), pp. 229-306. ${ }^{2}$ J. Richter, J. Schulenburg, and A. Honecker, Lect. Notes Phys. 645, 85 (2004).

\footnotetext{
${ }^{3}$ R. Moessner, Can. J. Phys. 79, 1283 (2001).

${ }^{4}$ P. Lecheminant, B. Bernu, C. Lhuillier, L. Pierre, and P. Sindzingre, Phys. Rev. B 56, 2521 (1997).

${ }^{5}$ Ch. Waldtmann, H.-U. Everts, B. Bernu, C. Lhuillier, P. Sindzin-
} 
gre, P. Lecheminant, and L. Pierre, Eur. Phys. J. B 2, 501 (1998).

${ }^{6}$ F. Mila, Phys. Rev. Lett. 81, 2356 (1998).

${ }^{7}$ C. Lhuillier, P. Sindzingre, and J.-B. Fouet, Can. J. Phys. 79, 1525 (2001).

${ }^{8}$ A. V. Syromyatnikov and S. V. Maleyev, Phys. Rev. B 66, 132408 (2002).

${ }^{9}$ P. Nikolic and T. Senthil, Phys. Rev. B 68, 214415 (2003).

${ }^{10}$ Z. Hiroi, M. Hanawa, N. Kobayashi, M. Nohara, H. Takagi, Y. Kato, and M. Takigawa, J. Phys. Soc. Jpn. 70, 3377 (2001).

${ }^{11}$ L. Santos, M. A. Baranov, J. I. Cirac, H.-U. Everts, H. Fehrmann, and M. Lewenstein, Phys. Rev. Lett. 93, 030601 (2004).

${ }^{12}$ M. E. Zhitomirsky, Phys. Rev. Lett. 88, 057204 (2002).

${ }^{13}$ A. Honecker, O. A. Petrenko, and M. E. Zhitomirsky, Physica B 312-313, 609 (2002).

${ }^{14}$ D. C. Cabra, M. D. Grynberg, P. C. W. Holdsworth, and P. Pujol, Phys. Rev. B 65, 094418 (2002).

${ }^{15}$ K. Hida, J. Phys. Soc. Jpn. 70, 3673 (2001).

${ }^{16}$ J. Schulenburg, A. Honecker, J. Schnack, J. Richter, and H.-J. Schmidt, Phys. Rev. Lett. 88, 167207 (2002).
${ }^{17}$ A. Honecker, J. Schulenburg, and J. Richter, J. Phys.: Condens. Matter 16, S749 (2004).

${ }^{18}$ A. Honecker, D. C. Cabra, M. D. Grynberg, P. C. W. Holdsworth, P. Pujol, J. Richter, D. Schmalfuß, and J. Schulenburg, condmat/0412608, Physica B (to be published).

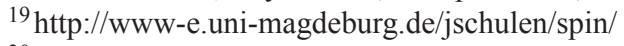

${ }^{20}$ J. T. Chalker, P. C. W. Holdsworth, and E. F. Shender, Phys. Rev. Lett. 68, 855 (1992); E. F. Shender and P. C. W. Holdsworth, in Fluctuations and Order: A New Synthesis, edited by M. M. Millonas (Springer-Verlag, 1996).

${ }^{21}$ R. Moessner, S. L. Sondhi, and P. Chandra, Phys. Rev. Lett. 84, 4457 (2000).

${ }^{22}$ R. Moessner and S. L. Sondhi, Phys. Rev. B 63, 224401 (2001).

${ }^{23}$ G. H. Wannier, Phys. Rev. 79, 357 (1950).

${ }^{24}$ F. Y. Wu, cond-mat/0303251 (unpublished).

${ }^{25}$ R. Moessner, S. L. Sondhi, and P. Chandra, Phys. Rev. B 64, 144416 (2001).

${ }^{26}$ One of these maps sends $s_{i, j}^{ \pm} \rightarrow-s_{i, j}^{ \pm}$with a suitable choice of one $j$ for each possible hexagon $i$ (R. Moessner, private communication). 